\title{
APPLICATIONS OF A REALISTIC MODEL FOR CCD IMAGING
}

\author{
Steve B. Howell and William J. Merline \\ Planetary Science Institute
}

\section{DESCRIPTION OF CCD MODEL}

We have constructed a computer model for simulation of point sources imaged on CCDs. An attempt has been made to ensure that the model produces "data" that mimic real data taken with two-D detectors. To be realistic, such simulations must include randomly generated noise of the appropriate type from all sources. The synthetic data are output as simple one-D integrations, as two-D radial slices, and as three-D intensity plots. Each noise source can be turned on or off so they can be studied independently as well as in combination to provide insight into the image components.

Some of the many properties of a detector and optical system that can be simulated include the effects of changing image position, image scale, signal strength, noise sources, and data reduction techniques. This model is useful for planning observational strategy and for optimization of photometric reductions. Details of the model, error analysis, software, and applications can be found in Merline and Howell (1992) and Merline and Howell (1995). Below, we discuss briefly some of our recent modeling endeavors.

\section{APPLICATIONS}

The Lowell Observatory Near-Earth-Object Survey (LONEOS), plans to use a 1-m Schmidt telescope with four 2048 x 2048 CCDs to survey 1000 sq. degrees of sky each night. The primary goal of the project is to detect numerous faint NEOs. We intend to use the LONEOS data for the first large-scale photometric search for extra-solar planets, by monitoring millions of stars per year for evidence of planetary transits across the stellar disks. While scintillation noise sets fundamental limits for a ground-based photometric search, we have used our model to show that transits by Jupiter- and Saturn-sized planets should be discernible in the light curves of the brighter late-type stars (see Howell 1995). Statistically, we expect one reliable transit observation each year.

NASA's first discovery class mission will be the Near-Earth-Asteroid Rendezvous (NEAR) Mission. The imager will consist of a 50-mm telescope and a frame transfer CCD. The CCD has rectangular pixels and the images will have substantial contributions from read and dark noise. These features will present challenges for calibration and image interpretation. We have used our model to perform some initial simulations for the NEAR imager (Fig. 1), as we had done previously for the Galileo imaging system. Typical targets for spacecraft instrument/filter calibration and pointing information are star clusters such as the Pleiades. 
Comet Shoemaker-Levy 9 presented the special problem of detecting faint signals against the bright backgrounds of Jupiter and its satellites. Our observational method used continuous readout of a CCD, yielding time-resolved imaging (one spatial dimension vs. time, resulting in streaked images) of Jupiter and its moons, high duty cycle (little dead time), and avoided saturation during the exposure, even on large-aperture telescopes. We applied our model to determine the optimum combinations of telescope aperture, image scale, detector, read rates, and filter. Because our model is intended for point-sources, modifications had to be made to simulate the scanned images, but this indicates the adaptability of the software.
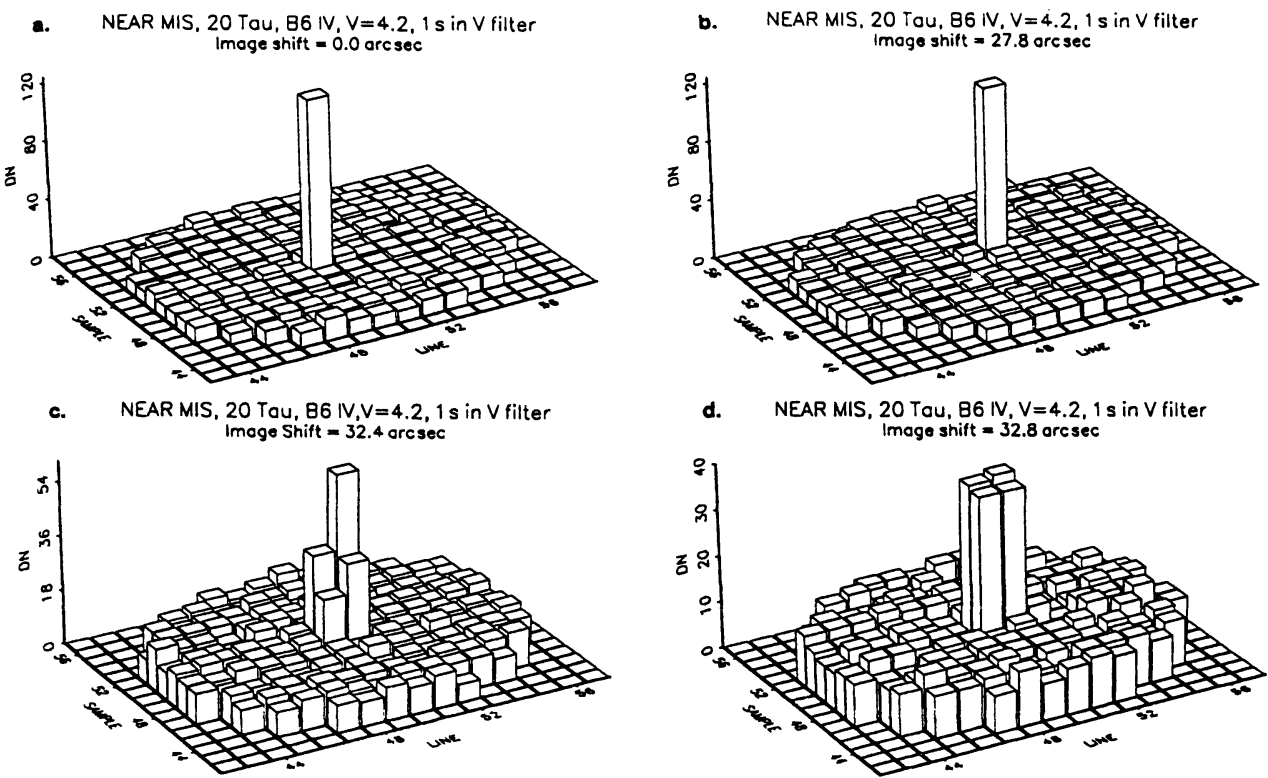

Fig. 1. Sample images of Pleiades star 20 Tau, seen by the NEAR imager, using our CCD modeling code. This demonstrates the sensitivity to positioning in undersampled (large pixels) images. Images $\mathrm{a}$ and $\mathrm{b}$ are indistinguishable, even though the image has been moved by nearly 28 arcsec. In model c, the image has been shifted an additional 4.6 arcsec, and in d only 0.4 arcsec more. A bias level of $10 \mathrm{DN}$ was arbitrarily chosen: pixels near the periphery are not calculated and are assigned value zero. This sensitivity to position will be important when using stellar images for navigation, optical distortion mapping, and in distinguishing possible asteroid satellites from cosmic rays.

\section{REFERENCES}

Howell, S. B. 1995, in Proc. IAU Symposium No. 167, New Developments in Array Technology and Applications, A. G. D. Philip, K. A. Janes and A. R. Upgren, eds., Kluwer Academic Pub., p. 167

Merline, W. J. and Howell, S. B. 1992 in Astronomical Data Analysis Software and Systems I, D. Worrall, C. Biemesderfer, and J. Barnes, eds., ASP Conf. Series, Vol 25, San Francisco, p. 316

Merline, W. J. and Howell, S. B. 1995 Experimental Astronomy, in press 